\title{
Survey of Metaheuristic Algorithms for Combinatorial Optimization
}

\author{
Malti Baghel \\ PG, Scholar, CSE Dept. \\ U.I.T., RGPV \\ Bhopal, India
}

\author{
Shikha Agrawal \\ CSE Department \\ U.I.T., RGPV \\ Bhopal, India
}

\author{
Sanjay Silakari Ph.D \\ CSE Department \\ U.I.T RGPV \\ Bhopal,India
}

\begin{abstract}
This paper is intended to give a review of metaheuristic and their application to combinatorial optimization problems. This paper comprises a snapshot of the rapid evolution of metaheuristic concepts, their convergence towards a unified framework and the richness of potential application in combinatorial optimization problems. Over the years, combinatorial optimization problems are gaining awareness of the researchers both in scientific as well as industrial world. This paper aims to present a brief survey of different metaheuristic algorithms for solving the combinatorial optimization problems. Basically we have divided the metaheuristic into three broad categories namely trajectory methods, population based methods and hybrid methods. Trajectory methods are those that deal with a single solution. These include simulated annealing, tabu search, variable neighborhood search and greedy randomized adaptive search procedure. Population based methods deal with a set of solutions. These include genetic algorithm, ant colony optimization and particle swarm optimization. Hybrid methods deal with the hybridization of single point search methods and population based methods. These are further categorized into five different types. Finally we conclude the paper by giving some issues which are needed to develop a well performed metaheuristic algorithm.
\end{abstract}

\section{Keywords}

Ant Colony optimization, Combinatorial optimization problems, Genetic algorithm, Greedy randomized adaptive search procedure, Particle swarm optimization, Simulated Annealing, Tabu search and variable neighborhood search.

\section{INTRODUCTION}

Optimization is an art of selecting the best alternative among a given set of options or it can be viewed as one of the major quantitative tools in network of decision making in which decisions have to be taken to optimize one or more objectives in some prescribed set of circumstances. Optimization problems arise in various disciplines such as engineering design, agricultural services, manufacturing system, economics etc. thus in view of the practical utility of optimization problems there is a need for efficient and robust computational algorithms which can numerically solve with computers the mathematical models of medium as well as large size optimization problems arising in different fields. Optimization problems can be of different type such as multi objective optimization, multimodal optimization and combinatorial optimization. In this paper, we discuss about combinatorial optimization. Many optimization problems of both theoretical and practical importance relates them with the selection of best configuration of a set of finite object to achieve certain goals. Such problems are basically divided into two categories one is those where solutions are programmed with real valued variable and the other is those where solution is programmed with discrete variable.
Combinatorial Optimization Problems are the one that belongs to the second category. A Combinatorial Optimization Problem (COP) can be stated as a finite set of possible solution from which we look for the best one minimum or maximum. These problems can be classified as NP-hard problems. According to [22]

A COP $\mathrm{P}=(S, f)$ can be defined by:

- A set of variables $X=\left\{\mathrm{x}_{1}, \ldots, \mathrm{x}_{\mathrm{n}}\right\}$

- Variable domains $D_{1}, \ldots, D_{n}$;

- Constraints among variables;

- An objective function $f$ to be minimized, where $f$ : $\mathrm{D}_{1} \mathrm{X} \cdots \mathrm{X} \mathrm{D}_{\mathrm{n}} \rightarrow \mathrm{IR}+$;

The set of all possible feasible assignments is:

$$
\mathrm{S}=\left\{\mathrm{s}=\left\{\left(\mathrm{x}_{1}, \mathrm{v}_{1}\right), \ldots,\left(\mathrm{x}_{\mathrm{n}}, \mathrm{v}_{\mathrm{n}}\right)\right\} \mid \mathrm{v}_{\mathrm{i}} \in \mathrm{D}_{\mathrm{i}}\right\}
$$

Since $s$ satisfies all the constraints. $S$ is usually called the search space, so that each set can be seen as a candidate solution. To solve a COP is necessary to find a solution $\mathrm{s}^{*} \in$ $\mathrm{S}$, such that the value of the objective function for $\mathrm{s}^{*}$ is the smallest possible (for a minimization function), which means, $\mathrm{f}\left(\mathrm{s}^{*}\right) \leq \mathrm{f}(\mathrm{s}) \forall \mathrm{s} \in \mathrm{S}$.

The rest of the paper is organized as follows: Section 2 presents an overview of Trajectory methods. This section is further subdivided into four subsections namely simulated annealing, tabu search, variable neighborhood search, greedy randomized adaptive search procedure. A literature review is given with each subsection. Section 3 presents an overview of Population based methods. This section is subdivided into three subsections namely genetic algorithm, ant colony optimization and particle swarm optimization. A literature review is given with each subsection. Section 4 presents hybrid methods. Hybrid methods are further divided into five subsections namely hybridizing metaheuristic with (meta-) heuristics, hybridizing metaheuristic with constraint programming, hybridizing metaheuristic with tree search technique, hybridizing metaheuristic with problem relaxation, hybridizing metaheuristic with dynamic programming. A literature review is given with each subsection and conclusion is reported in Section 5

\section{TRAJECTORY METHODS}

In this part, we outline Metaheuristics called as Trajectory methods. Trajectory methods are those working on a single solution. The term trajectory methods are used because searching done by these methods is characterized by a trajectory in the search space. The characteristics of the trajectory methods provide knowledge about the performance of the algorithm and its potency with respect to the instance that is tackled. The following subsections will provide an introduction and survey of various types of trajectory methods. 


\subsection{Simulated Annealing}

Simulated annealing [64] was first proposed by Kirkpatrick et al. in 1983. It is a randomized local search algorithm that had an explicit strategy to escape from local minima. The algorithm is analogous to the physical annealing process. Modification to the current solution results in an increasing solution cost that can be accepted with some probability. Iteratively, modification to the current solution is done by randomly selecting a move from the neighborhood. If it is an improving solution, then it is automatically accepted as a new current solution otherwise, it will be accepted (according to the Boltzmann distribution) with certain probability using a control parameter called the Temperature. When the temperature is high and the cost increase is low then the move may accepted with certain probability. According to predefined cooling schedule, the temperature is increasing turned down. When the temperature is adequately low, then method stops at a local optimum by allowing only improving moves.

David T. Connolly (1990) proposed an improved simulated annealing for solving quadratic assignment problem. The experiment shows that it is a much improved annealing scheme for this problem, finding better solutions with less amount of computational effort [32]. Laarhoven et al. (1992) proposed simulated annealing for solving the job shop scheduling problem. The proposed approach involves the acceptance of cost increasing transitions with a non zero probability to avoid being trapped in local minima [95]. Alfonsas (2003) proposed a modified simulated annealing for solving quadratic assignment problem. The proposed approach contains an advanced formula for the computation of initial and final temperature and also cooling schedule with oscillation. In order to improve the performance simulated annealing is merged with a tabu search algorithm [4]. Huajin jang et al (2006) proposed a simulated annealing for solving traveling salesman problem. In this a new Columnar Competitive Model (CCM) is introduced with the winnertakes-all (WTA) learning rule for solving the combinatorial optimization problem [39]. A.Al-khedhairi (2008) proposed simulated annealing for solving p-median problem. The purpose is to find the optimal (or near optimal) solution of the P-median problem [3]. In (2009) Jingfa liu et al. proposed a heuristic simulated annealing algorithm to solve the circular packing problem. The proposed Heuristic Simulated Annealing (HSA) algorithm includes a heuristic neighborhood search mechanism and the gradient descent method into the simulated annealing method. The neighborhood search mechanism can avoid the shortcomings of blind search in the simulated annealing and the gradient descent method can stimulate searching the global optimal configuration [62]. Xu Hao (2010) proposed a heuristic algorithm for solving traveling salesman problem. The approach involves the crossover and mutation operator to achieve a balance between speed and accuracy. Experiment shows that algorithm is better than the traditional method [117].

\subsection{Tabu Search}

Tabu search [41] was first proposed by Glover in 1986. Tabu search (TS) is a metaheuristic that guides a local search heuristic both to escape from local minima and to implement an exploration scheme. The simple TS algorithm applies a best improvement local search where at each iteration the best solution in the neighborhood is selected as the new current solution. A short-term memory is implemented as a tabu list where solution attributes are stored to avoid short term cycling. A term Aspiration criteria are defined which is allowed to include some unvisited solution of good quality which are excluded from allowed set.

Starting from the basic search strategy a number of developments and elaboration have been proposed over the years. These include Introduction of intensification and diversification mechanisms. They are implemented via different forms of long term memories. Adaptive memories provides a mechanism to diversity and intensity of the search so as to create more flexible search behaviors. Frequency memories provides a form of continuous diversity by introducing a bias in the evaluation of neighborhood solution. The probabilistic TS introduce randomization into the search method. It can be attained by linking probabilities with moves contributing to the neighborhood solution. Unified tabu search is aimed at developing simpler, more flexible TS code another example of this style is the granular TS.

Laguna et al. (1991) proposed tabu search methods for a single machine scheduling problem. The approach consists of the use of three local search methods within a tabu search to solve the problem. Also a hybrid TS is developed that utilize both swap and insert moves [71]. Barnes et al. (1993) proposes static TC for solving the multiple mechanic weighted flow time problem. The proposed approach provides superior result in comparison to the Branch and Bound method [12]. Battiti et al. (1994) introduced the reactive search which uses the history of the search for dynamically adjusting search parameters. In particular, when configurations are frequently occurring the dimensions of tabu list are automatically increased to avoid short term cycle [14]. Laguna et al. (1995) applied a TS method to solve the multilevel generalized assignment problem in which at each iteration, list of candidate moves are generated by using ejection chains [70]. Carlton and Barnes (1996) applied reactive TS to solve Travelling Salesman problem. In this a robust tabu search is applied to traveling salesman problem with time window. The approach uses a two level hashing method within a reactive tabu search to find unique repeated solution and to upgrade a more diverse search [25]. Lokketangen et al. (1998) applied TS to solve general zeroone mixed integer programming problem. In this specialized choice rule and aspiration criteria are discovered for the problem, expressed as a function of integer that measures infeasibility and objective function values [74]. Nanry et al. (2000) applied reactive TS to solve a pick up and delivery problem with the constraint of vehicle capacity and customer time windows [88]. Gonzalez-Velarde et al. (2002) applied TS to solve graph coloring problem. In this paper, a tabu search method is proposed that applies a simple version of ejection chains for coloring graph [48]. Korycinski et al. (2003) merged TS within a classification algorithm using reactive TS to improve classification accuracy [65]. Scrich et al. (2004) applied TS to solve the problem of scheduling job with the aim of minimizing total tardiness [107]. Barnes et al. (2004) applied TS to solve the aerial fleet refueling using group theoretic. They applied group theory to partitioning and ordering combinatorial optimization [13]. Harwig et al. (2006) applied adaptive TS to solve two dimensional orthogonal packing problems [51]. Kinney et al. (2007) proposed a group theoretic TS algorithm to solve the unicost set covering problem by partitioning the solution space into orbits. In this a linear programming relaxation of the problem is solved and a quality solution profile is constructed by using linear programming optimization. Problem variables will be clustered based on this profile and partition the solution space into orbits [63]. James et al. (2009) showed the advantages of applying strategic diversification within Tabu search (TS) 
model for the quadratic assignment problem by including many diversification and multi start TS variants [61]. Ulrike (2011) applied TS to Scheduling problem then with the help of intensification and diversification strategies demonstrates that the result achieved is quite better [115].

\subsection{Greedy Randomized Adaptive Search Procedure}

Greedy randomized adaptive search procedure (GRASP) was introduced by Resende in 1995. GRASP [44] is a multi start or iterative procedure consisting of 2 phases. One is Construction phase and another is a local search phase. The construction phase creates a feasible solution by applying a greedy randomized criterion which is then improved until a local minimum is found during the local search phase the best overall solution is returned as the result. Construction phase can be illustrated by 2 basic types. One is Dynamic constructive heuristic and other one is Randomization. In constructive heuristic the elements that are not included in the partial solution are estimated with a greedy function and the list of best elements are kept in a so called restricted candidate list (RCL). Through randomization any element is randomly chosen and included in the partial solution thus resulting in a diversity of solutions. GRASP is memory less algorithm.

Atkinson et al. (1998) applied GRASP to time constrained vehicle scheduling here two forms of adaptive search called local and global adaptation was described [8]. Fleurent et al. (1999) applied GRASP to solve quadratic assignment problem using Adaptive memory [45]. Laguna et al. (1999) introduces GRASP with path relinking to improve performance [72]. Prais et al. (2000) introduces a Reactive GRASP for a matrix decomposition problem in TDMA traffic assignment. In this work author suggested the refinement of GRASP that self adjusted the parameter values instead of fixed values in the construction phase [98]. Binato et al. (2001) introduces a new metaheuristic approach called greedy randomized adaptive path relinking (GRAPR). It uses generalized GRASP concepts in path relinking to explore different trajectories between two good solutions previously found [16]. Ribeiro et al. (2002) proposed a hybrid GRASP to solve steiner problems in graphs using weight perturbation and adaptive path relinking heuristic (HGP-PR) [103]. Hirsch et al. (2007) introduced continuous GRASP (C-GRASP) by extending GRASP from discrete optimization to continuous global optimization [52]. Andrade et al. (2007) proposed GRASP with an evolutionary path relinking to solve the network migration problem. The authors proposed a metaheuristic resulting from the hybridization of GRASP, path relinking, evolutionary path relinking [5]. Arnaldo and Rafael (2010) applied GRASP heuristic, merged with a path relinking for solving school timetabling problem [6]. Yannis (2012) proposed an enhanced version of GRASP called Multiple Phase Neighborhood Search GRASP (MPNS-GRASP) for solving vehicle routing problem. In this method, a stopping criterion based on Lagrangean relaxation and sub gradient optimization is applied. Also, another strategy known as the Circle Restricted Local Search Moves strategy was used for expanding the neighborhood search [118]

\subsection{Variable Neighborhood Search}

Variable neighborhood search (VNS) was proposed by Mladenovic and Hansen in 1997. VNS [86] is a method based on the idea of dynamically changing neighborhood within a local search.VNS proceeds by a descent method to get from the neighbors to local optima exploring, systematically or at a random distant neighborhood of the incumbent solution. The method moves from this current solution to new one if there is an improvement in the solution. In this way, optimal variable is kept in the incumbent and obtains promising neighbors. Therefore VNS is not a trajectory following method. VNS main cycle consist of three phases. Shaking, local search and move. Basically VNS is extended into many forms. They are Variable Neighbor Descent, Reduced VNS, Basic VNS, Skewed VNS, General VNS, VNS Decomposition search and many more.

P. Hansen and Mladenovic (1997) introduced VNS for PMedian problem. Experiment shows that the proposed variable neighborhood search heuristic is quite better than other heuristics [50]. A.S.Wade and V. J. Rayward Smith (2000) proposed VNS for solving Steiner tree problem by using an effective local search technique [116]. In 2001, N. Mladenovic and Urosevic proposed VNS for solving $\mathrm{K}$ cardinality tree problem [87]. Ribeiro and Souza (2002) proposed a VNS for solving degree constrained minimum spanning tree problem. Here VNS heuristic is proposed which is based on dynamic neighborhood and a variable neighborhood descent iterative improvement algorithm is used for local search [21]. Avanthay at al. (2003) introduced an adaptation of the VNS method to graph coloring problem. The proposed technique has proved to be effective in determining high quality solutions [18]. Puchinger and Raidl (2005) applied VNS to Multidimensional knapsack problem. Here a variant of VNS called Relaxation guided VNS is introduced. It is based on the general VNS scheme and a new variable neighborhood descent algorithm [99]. Sevkli and Aydin (2006) proposed VNS for the job shop scheduling problem. The idea is to construct the best local search and shake operations based on neighborhood structure [108]. Consoli et al. (2009) proposed a VNS for minimum labeling Steiner tree problem. In this paper, a hybrid local search method known as Group-Swap Variable Neighborhood Search (GSVNS) is proposed. It is a hybrid of two metaheuristics named Variable Neighborhood Search and simulated Annealing. Experiment illustrates that the proposed heuristic quickly gives optimal or near optimal solutions [30]. Mladenovic et al. (2010) proposed a Variable neighborhood search for the matrix bandwidth minimization problem. In this paper, a VNS based heuristic is introduced which provide high quality solution to the problem [85]. Geiger et al. (2011) proposed neighborhood selection in VNS for solving single machine total weight tardiness problem [83]. Duarte et al. (2012) introduces a pure 0-1 optimization model and a metaheuristic algorithm based on the VNS methodology for the vertex separation problem on general graphs [36].

Other trajectory methods include Basic local search, Iterated local search, Guided local search. These can be found in [7]

\section{POPULATION BASED METAHEURISTICS}

This type of Metaheuristics explicitly works with a set of solution .In this, a different solution is merged, either implicitly or explicitly, to create new solutions. The methods are as follows.

\subsection{Genetic Algorithms}

Genetic algorithm was developed by John Holland in the 1960 's. Genetic algorithms [56] are a class of adaptive search procedure. They are based on the principle of evolution via natural selection act on a population of individuals that undergo selection in the presence of operators such as mutation and crossover. A fitness function is used to calculate individuals and reproductive success diverge with fitness. 
Genetic algorithm uses evolutionary techniques to solve a solution of different problems. The basic step of a genetic algorithm is simple. At each iteration, a population of solution is evolved from one generation to the next generation. Next the better solutions are recombined with each other to form new solutions. Finally the new solutions generated will replace the poorest of the original solution and the process is repeated.

Tate et al. (1995) proposed a genetic algorithm to solve quadratic assignment problem. Experiment illustrates that genetic algorithms find a better solution than those of the best previously known heuristics [113]. Chu and Beasley (1996) proposed genetic algorithm to solve the set partitioning problem. In this paper basic genetic algorithm components such as fitness definition, parent selection and population replacement are modified. Experiment shows that genetic algorithm based heuristic is producing high quality solutions [55]. Chu and Beasley (1997) proposed a genetic algorithm based heuristic for solving generalized assignment problem. In this approach, GA heuristic includes a problem-specific coding of a solution structure, a fitness-unfitness pair evaluation function and a local improvement procedure. Experiment shows that the genetic algorithm heuristic is able to find high quality solutions [94]. Chu and Beasley (1998) proposed genetic algorithm for solving multidimensional knapsack problem. In this approach, a heuristic based upon genetic algorithm is introduced. The proposed heuristic is capable of obtaining high quality solutions to the problem [28]. Ahuja et al. (2000) proposed genetic algorithm to solve the quadratic assignment problem. In this approach genetic algorithm includes many greedy principles in its design for solving the problem [100]. Olli Braijsy (2001) proposed genetic algorithm to solve vehicle routing problem with time window. In this paper, three evolutionary algorithms were described and compared with the other metaheuristic algorithm. Experiment shows genetic algorithm is not competitive with the best published result though the differences are not overwhelming [90]. Chiung Moon et al. (2002) proposed a genetic algorithm to solve traveling salesman problem with precedence constraints. In this paper, the key concept is the topological sort. Also a new crossover operation is introduced for the proposed genetic algorithm .Experiment shows that the proposed genetic algorithm produces an optimal solution and shows better performance compared to the traditional algorithm [27]. Omar et al. (2009) proposed an improved genetic algorithm to solve traveling salesman problem. In this approach the new crossover operation, population reformulates operation, multi mutation operation, partial local optimal mutation operation and rearrangement operation are used to solve the problem [91]. Tamura et al. (2010) proposed an improved genetic algorithm for solving scheduling problem. In this, the proposed approach is improved by a genetic algorithm using multipliers can be altered during the search process [92]. Kusum and Hadush (2011) proposed a genetic algorithm for solving traveling salesman problem. In this, two mutation operations named inverted exchange and inverted displacement are combined to increase the performance of genetic algorithm [68]. Neelam et al. (2012) proposed genetic algorithm to solve flow shop scheduling problem. In this, a genetic algorithm based heuristic is described to make span minimization on flow shop scheduling [89].

\subsection{Ant Colony Optimization}

Ant colony optimization (ACO) was proposed by Dorigo in1991, inspired by the foraging behavior of real ants [35]. Ants can find the shortest path from the nest to food source through a chemical substance called pheromone. As ants move, a certain amount of pheromone is layered on the ground making a pheromone trail. As the ants follow the trail, they will be influenced by the trail even more. This process involves a positive reinforcement loop in which the probability that ants will visit the path in the future is directly proportional to the number of ants that previously visited it. It has been proved experimentally that this procedure can give rise to growth of shortest paths. Here, at each cycle, solution is created in a randomized and greedy way by a number of artificial ants. However, Heuristic evaluation and the amount of pheromone are the one by which an individual ant chooses the next element to be included into current partial solution. When a complete solution is built, the procedure is restarted with the updated pheromone levels. This is repeated for a fixed amount of cycles or until search stagnation occurs.

M. Dorigo et al (1994) proposed a new heuristic called ant system for solving job shop scheduling problem. In this, search task is spread over many simple, loosely interacting agents which can be used to find good solutions [29]. Dorigo and Gambardella (1997) proposed a distributed algorithm ant colony system for solving traveling salesman problem. In this, a group of agents cooperates using an indirect form of communication to find the best solution to the traveling salesman problem [34]. Thomas (1998) proposed ACO based on Max-Min ant system to solve flow shop problem. MaxMin ant system is enhanced by a fast local search procedure to produce high quality solutions to the problem [114]. Bullnheimer et al. (1999) proposed an ant system for solving vehicle routing problem. An ant system is a distributed metaheuristic that merges an adaptive memory with a local heuristic function to frequently construct solutions of the problems [17]. Talbi et al. (2001) proposed a parallel model of ACO to solve the quadratic assignment problem. The parallel ant colony optimization algorithm has been merged with a local search method that depends on a tabu search [40]. Bell and Mcmullen (2004) proposed an ant colony optimization technique for the vehicle routing problem. It has been proved experimentally that the algorithm is successful in finding a competitive solution technique especially for larger problems [15]. Neumann and Witt (2006) proposed an ant colony optimization for minimum spanning tree problem. Solution to the problem is built by a random walk on a so called construction graph. This random walk can be determined by the heuristic information [46]. Yu bin et al. (2009) proposes an ant colony optimization for solving the vehicle routing problem. The proposed algorithm owns a new scheme by modifying the increased pheromone called ant weight strategy and using a mutation operator to solve the vehicle routing problem [2]. Sureka et al. (2010) proposed an ant colony optimization for solving job shop scheduling problem. In this, fuzzy logic is used for scheduling the sequence of job and it can be optimized using ACO [110]. Zar chi Su Su Hlaing and May Aye Khine (2011) proposed an ACO for solving travelling salesman problem in which, good distribution strategy and information entropy is conducted. Also, it uses local optimization heuristic for solving traveling salesman problem along with the basic ACO and the proposed algorithm shows better performance than ACO algorithm [119]. 


\subsection{Particle Swarm Optimization}

Particle Swarm Optimization (PSO) was proposed by Eberhart and Kennedy in 1995. PSO [57] is a computational optimization technique which is inspired by the social behavior of bird flocking. PSO is a searching technique utilizes a group of particles that move through the search space to find the global minimum. In PSO, a group of particles is initialized randomly. Each particle represents a possible solution and then iteratively finds the optimal solution. There are basically two extremes called as local and global extreme. Fitness value is calculated. If it is better than it is set as new local best. Now choose the particle with the best fitness value of all particles as the global best. Finally each particle velocity and particle position is calculated and updated.

Ayed Salman et al. (2002) proposed PSO to solve task assignment problem. In this new task assignment algorithm is presented which is based on the principle of PSO. The effectiveness of the proposed PSO based algorithm is demonstrated by comparing it with another population based heuristic known as genetic algorithm. Experiment shows that PSO based algorithm is a viable approach for traveling salesman problem [9]. Kang-Ping Wang et al. (2003) proposed PSO to solve traveling salesman problem. In this paper, the author proposed the concept of swap operator and swaps sequence and redefined some operators on the basis of them. Experiment shows that result achieved are quite better [66]. Tasgetiren et al. (2004) proposed PSO to solve the single machine total weighted tardiness problem. This paper embedded an efficient local search method and a heuristic rule known as Smallest Position Value (SPV) to the continuous PSO for solving sequencing problem [112]. Chen et al. (2006) proposed a hybrid PSO algorithm to solve the capacitated vehicle routing problem. In this, discrete PSO is used which merges the global search and local search to achieve an optimal solution and also simulated annealing is used to avoid being trapped in local minimum [26]. Yuan and Zhao (2007) proposed a modified version of binary PSO that uses Smallest Position Value (SPV) heuristic rule to solve permutation flow shop problem. Also, local search and perturbation is used to improve the convergence speed of the algorithm [73]. Guner and Sevkli (2008) proposed a discrete PSO for solving the uncapacitated facility location problem. Here a local search is used to provide more efficient result [109]. Consoli et al. (2010) proposed discrete PSO for solving the minimum labeling steiner tree problem. In this paper a discrete PSO called Jumping Particle Swarm Optimization (JPSO) is introduced. JPSO is compared with another algorithm and found that JPSO outperformed all other algorithm achieving high quality solutions in fewer amounts of computational efforts [31]. Rosendo (2010) proposed a hybrid PSO algorithm to solve the traveling salesman problem. The hybrid approach uses the concept of local search and path relinking [84]. Pierobom et al. (2011) proposed to solve the task assignment problem. In these two versions are used, one is based on binary codification and other uses permutation to encode the solution. The experiment shows that the permutation encoding is better than the binary encoding [97]. Kunpeng Kang (2012) proposed a fast PSO algorithm for solving large scale multidimensional knapsack problem (MKP). In this paper, a strategy of mimetic Profit/Weight ratio (P/W-Rt) is introduced which can effectively solve MKP with arbitrary number of constraints under same computational cost as one dimensional knapsack problem. Experiment shows the efficient and robust performance of the proposed algorithm. Another advantage of the proposed algorithm is that it is quite flexible to the size of the MKP instance [67].

\section{HYBRID METAHEURISTICS}

Over the years, it has become apparent that the need of single traditional metaheuristic has been restricted on the contrary, they are merged with other optimization algorithm called as Hybrid Metaheuristics. The inspiration for hybridization of different algorithm is to accomplish the interrelated behavior of different optimization strategies. There have been following type of hybridization taken place. Hybrid metaheuristic can also be referred from [19].

\subsection{Hybridizing Metaheuristics With (Meta-) Heuristics}

Today researchers are combining one metaheuristic with another (meta-) heuristic for optimization. Basically, different combination can be formed by their merging. One can be the combination of local search methods and population based method. Population based methods like an ant colony optimization and evolutionary computation make the use of local search methods to provide high quality solutions by processing the solution. Briefly saying population based method to determine the promising extent of the search space where local search can then find the best solution rapidly. The hybridization of this type is very successful. The term Memetic algorithm is derived from the hybridization of a genetic algorithm (or evolution algorithm) with the non genetic local search algorithms. Many examples were proposed by several researchers based on this type of hybridization.

Walshaw et al. (2004) proposed multilevel technique to Combinatorial Optimization problems. A multilevel method is an efficient and effective algorithm for solving large scale computational and optimization problem. In multilevel technique, original problem is taken as input; a hierarchy of problems is created by creating smaller instances of problem by successive coarsening until conditions are met. Then optimization technique can be used to create solution to the smallest problem. Then this solution would be transformed to the next level until the solution to the original problem is acquired [23]. Another iterated local search algorithm Population based iterated local search (PBILS) was introduced by Thomas (2006) .A population of solution is used for extracting the useful information which is combined with iterated local search. In order to store their common substructure, where the current solution population member disagree, PBILS keeps small population and enclose the perturbation of iterated local search to subspace. The key postulation of PBILS is that local optimal solution owns common substructure that can be used to enhance the efficiency [111]. Besides the two, there are many more techniques of hybridization between metaheuristic and other (meta-) heuristics that was proposed by several researchers. Lozano et al. (2010) proposed a hybrid metaheuristic that contain iterated local search algorithm that uses an evolutionary algorithm as a perturbation technique. The proposed work helps to deal with binary optimization problems [78]. Another work include of Resende et al. (2010) proposed several variants of a hybrid algorithm which is a combination of GRASP and path relinking. The hybridization is carried for maximum minimum diversity problem [82]. Another work proposed by Hanafi et al. is a Variable neighborhood decomposition approach (2010) which hybridizes VNS with general purpose CPLEX MIP solver. The hybridization was done to solve $0-1$ mixed integer 
program [58]. Glover and Laguna proposed a concept based on the tabu search for improving metaheuristic that is called as a proximate optimality principle (POP). The key assumption of this work is that good solutions have parts in common and they will appear close to each other in the search space [43]. Montemanni and Smith (2010) proposed an improved tabu search for solving frequency assignment problem. Hereby, tabu search is enhanced by heuristic manipulation, which is based on adding constraints to a problem so as to reduce the search [101].

\subsection{Hybridizing Metaheuristic With Constraint Programming}

Metaheuristics and constraint programming are two different techniques of optimization. In constraint programming, constrains optimization problem can be either mathematically or symbolic simulated by means of variables, domains and constraints. Constraints summarize the distinct part of problems into sub problems. Each constraint is related to filtering algorithm that remove values which do not put in for feasible solutions. The solutions process of $\mathrm{CP}$ has two phases. One is propagation phase and another is labeling phase. In case of break down the search back track. When an optimization problem is solved a bound constraint is carried by placing an improving solution every time. Metaheuristics are efficient in fining a high quality solution but they are not very strong to solve constraint satisfaction problem on the other side CP is extremely efficient in solving decision problems but it is inadequate to tackle an optimization problem.

Shaw (1998) proposed a hybrid algorithm containing constraints programming and local search method to solve vehicle routing problem. Basically the approach is to explore a large neighborhood of current solutions by choosing a number of customer visits to take away from routing plan and inserting them using a constraint based tree search [93]. Meyer proposed the hybridization of Ant Colony Optimization and constraint programming to solve machine scheduling problem. Hybridization includes the technique for limiting the search performed by an ACO algorithm to promising realms of the search space [11]. Another prominent example of the incorporation of metaheuristic and constraint programming was proposed by J. C. Beck (2007) as solution guided multipoint construction search. It is a novel structural search approach that achieved a sequence of resource limited tree searches where search start either from the vacant solution or from the solution that come across the search some no of selected solution is selected during the search [59]. Khichane et al. (2008) proposed a hybrid algorithm that includes ACO and constraint programming to solve car sequencing problem. In this approach, the goal is to find any complete assignment satisfying all the constraints [76]. Prestwich et al. (2009) proposed the integration of evolutionary computation and neural network with constraint programming which result in a hybrid stochastic constraint programming structure that aims to find a policy tree which indicate the decision assignment at any possible situation [105].

\subsection{Hybridizing Metaheuristic With Tree Search Technique}

The hybridization of metaheuristic with the tree search technique is one of the popular ways of combining different algorithm for optimization. It is because many metaheuristic as well as some the major complete algorithm belongs to group of tree search technique. Tree search technique views the search space in the form of a tree. As constraints programming is used for exploring large neighborhood similarly there is a tree search method which can be applied to the same mainly branch and bound technique depend on linear programming, including branch and cut shows potential when the problem can be expressed by a mixed integer programming [MIP] model.

One of the first works on a hybridization of branch and bound with an evolutionary algorithm is done by Nagar et al. (1995) for a two machine flow shop scheduling problem [1]. Applegate et al. (1998) proposed tree search method to solve traveling salesman problem. Experiment shows that better solutions are generated by merging solution so as to provide optimality [33]. Another proposed by Shi and Olafsson (2000) where breadth first searched is hybridized with backtracking for nested partitioning which is used to explore the search space under the guidance of a metaheuristic. The work mentioned above is qualified by a subsidiary use of the tree search method within metaheuristic [69]. Danna et al. (2003) proposed an integration of mixed integer programming and local search to solve job shop scheduling problem. In this, metaheuristic can be used as subsidiary technique known as diving where the search processed is focused on neighborhood to provide high quality solutions [37]. Rothberg (2007) proposed a hybridization of an evolutionary algorithm in a MIP solver found on a branch and cut. The approach can be used for finding the best solution for different MIPs [39]. Another work proposed by Gallardo et al. (2007) in which flow of beam search and memetic algorithm are intertwined [60]. A fruitful problem specific paradigm for large neighborhood search has been proposed by Prandtstetter and Raidl (2008) for solving the car sequencing problem [79]. C. Blum et al. (2010) proposed Beam ACO that includes ACO and beam search for solving traveling salesman problem with time window [77].

\subsection{Hybridizing Metaheuristics With Problem Relaxation}

Taking metaheuristic problem relaxation has become a successful hybrid approach in recent year. A so- called relaxed version of the problem is achieved by modifying or recovering constraints from the original problem. The desire is to achieve two things. First one is that the relaxed problem can be solved well and the other is the construction of an optimal solution to the relaxed problem can be used to solve the original problem simultaneously with its objective function. An important kind of relaxation in combinatorial optimization relates by metaheuristic the integrity constraints of the involved variable. The leading linear programming relaxation can be efficiently solved by method like such as simplex algorithm to optimality.

A straightforward way to optimality is to derive a heuristic integer solution such that it can be feasible for the main problem. It can be simple rounding or more sophisticated repairing strategies depend on the problem. Tamura et al. (1994) propose an algorithm for job-shop scheduling problem that work by first implementing a genetic algorithm identifying a promising area of the search space then the fitness of the solution is calculated by Lagrangian relaxation. Then, exhaustive search is performed to find the best solution within the search space [49]. Another work proposed by Chu and Beasley (1998) for the multidimensional knapsack problem is an evolutionary algorithm that use dual variable values for calculating pseudo utility ratios for the variable [96]. Raidl and Feltl (2004) proposed a hybrid genetic algorithm for the generalized assignment problem. The LP 
relaxation is used to create an initial population of integral solution by a randomized rounding procedure [47]. Vasquez and Vimont (2005) proposed a two phase algorithm for 0-1 knapsack problem. In the first phase by neglecting the integrity constraints, a number of relaxed problems can be solved to optimality. In the second phase, tabu search is enforced to search to around the best possible solution to these relaxed problems [81]. Another work reported by Haouari and Siala (2006) presents the hybrid Lagrangian genetic algorithm for prize collecting steiner tree problem. Hereby genetic algorithm makes use of a Lagrangian relaxation. Meaningful initial solutions are generated by reducing the graph by cutting edge and the objective function is modified by cost reduction [75]. For the knapsack constrained maximum spanning tree problem, [104] a hybridization of Lagrangian decomposition and a genetic algorithm is proposed by Pirkwieser et al. (2007). Finally an ant colony optimization for the symmetric TSP is proposed by Reimann (2007) where optimal solution is used for prejudicing the search of the artificial ants towards the edges of the minimum spanning tree [80].

\subsection{Hybridization Metaheuristic With Dynamic Programming}

Dynamic programming (DP) is an example of an optimization method that can be successfully combined with metaheuristic, both in the case of constructive and local search technique. Iterated dynasearch are a hybrid metaheuristic in which DP is used as a neighborhood exploration approach inside iterated local search. The motivation behind this integration is that in neighborhood exploration strategy, the greater the neighborhood size more effectively the quality of the local optimum generates. In some cases in polynomial time, DP can make it possible to completely explore an exponential size neighborhood.

Congram et al. proposed iterated dynasearch (2002) used to solve for single machine total weight tardiness scheduling problem. It is the problem of discovering the processing order of many jobs on single machine such that the total tardiness is minimized [102]. Balakrishnan et al. (2003) proposed a hybrid algorithm that contains evolutionary algorithms and DP for solving dynamic facility layout problem. Here several solutions are given, DP is used as a crossover operator for determining the best combination for different planning period [53]. Tse et al. (2007) proposes a technique called an iterative dynamic programming that combine GA with local search technique. Here the problems are subdivided into sub problems and then DP optimizes separately [106]. Hu and Raidl (2008) proposed a hybrid algorithm for solving generalized traveling salesman problem. The authors use DP in the context of an evolutionary algorithm as a method for generating the optimal solution that can be generated from an incomplete solution [10]. Wilbaut et al. (2009) proposes a hybrid method merging adaptive memory, sparse DP and reduction technique to explore and reduce the search space. Here initially, bi-partition of the variable are created then the resulting small problem is solved and the space of the remaining variable is searched using tabu search [24]. Another work proposed by Bautista et al. (2009) use a heuristic version of DP labeled bounded dynamic programming for simple assembly line balancing problem. Here at each level number of positions is heuristically reduced leading to the optimal solution in a reduce amount of time [54]. Blum and Blesa (2009) propose the use of DP algorithm is two different metaheuristic for the K-cardinality tree (KCT) problem. The basic idea of this strategy is not only applicable to KCT problem but also for another subset problem. Basically, the plan is to generate a large number of objects then a solution is found by applying metaheuristic. These objects carry an exponential number of solutions to the problem and DP is used to find best solution efficiently for each object [19].

\section{CONCLUSIONS}

In this article we have provided a survey on the different techniques of metaheuristic for combinatorial optimization. We divided this growing research area into three broad categories named trajectory method, population based method and hybrid method. Trajectory methods are effective because they give knowledge about the behavior of the algorithm and its dynamic nature helps in selecting the most efficient method to solve the problem. Population based methods provide a natural way for the exploration of the search as it deals with the set of solutions. Hybrid methods include the integration of various metaheuristic algorithms which are efficient in finding good solutions that cannot be obtained by any complete method within a feasible time.

Finally it is concluded that before developing a metaheuristic, researcher has to go through some issues like the metaheuristic they are developing work for their problem or not. For example, for simulated annealing, tabu search, variable neighborhood search, a central issue is the design of the appropriate neighborhood structure. Greedy randomized adaptive search procedure uses a classical randomized greedy insertion heuristic to construct a solution followed by local search. Similarly genetic algorithm implementations use the integer string representation to encode a solution. Some common issues include are memory, randomization, dynamic parameter adjustment, large scale neighborhood scale. A fundamental element of metaheuristic is the use of memories to guide the search. It is a way of providing mechanism based on the history of the search process. Memories have been associated with tabu search, simulated annealing, ant colony optimization also now recent implementations of metaheuristics like VNS and GRASP include memories. Another issue concerns the use of randomization. Randomization provides a better way for the exploration of the search space by supporting a creation of diverse solutions. Randomization is inherently present in many metaheuristic like simulated annealing, ACO and GRASP. Another issue includes dynamic parameter adjustment. In this, parameters are self adjusted during the search process and providing robustness to the metaheuristic. Another important issue includes the large scale neighborhood structure. These allow a more thorough search of the solution space. These large neighborhoods are joined with some filtering techniques that aimed at focusing the search on most interesting solutions and neglecting others as considering the entire neighborhood evaluation would be extremely costly. These structures provide optimal solution for complex combinatorial optimization problems. They also provide simplicity, flexibility and robustness to the algorithm. The process of designing and implementing a metaheuristic is not an easy task it involves deep knowledge about an extensive range of algorithmic techniques, coding and data structure as well as algorithm engineering and statistics. For developing a well performed algorithm researchers need to know a literature search for developing a metaheuristic for the problem at hand. 


\section{REFERENCES}

[1] A. Nagar, S.S. Heragu \& J. Haddock. 1995. A metaheuristic algorithm for a bi-criteria scheduling problem. Ann. Oper. Res. Vol. 63, pp. 397-414.

[2] A. Yu Bin, Y. Zhong-Zhen \& Y. Baozhen. 2009. An improved ant colony optimization for vehicle routing problem. Eur. J. Oper. Res. Vol. 196, pp.171-176.

[3] A. Al-khedhairi.2008. Simulated annealing metaheuristic for solving P-median problem. Int. J. Contemp. Math. Sciences vol.3. pp.1357-1365.

[4] Alfonsas Misevicius.2003. A modified simulated annealing algorithm for the quadratic assignment problem. Informatica.vol.14, pp.497-514.

[5] Andrade, D.V., Resende \& M.G.C. 2007. GRASP with evolutionary path-relinking. Proceedings of The Seventh Metaheuristics International Conference.

[6] Arnaldo Vieira Moura, Rafael Augusto Scaraficci. 2010. A grasp strategy for a more constrained school timetabling problem. Int. J. Oper. Res. Vol. 7, pp.152170.

[7] A. Roli and C.Blum. 2003. Metaheuristics in combinatorial optimization: overview and conceptual comparison. ACM Computing Surveys.vol. 35 pp. 268308.

[8] Atkinson, J.B .1998. A greedy randomized search heuristic for time-constrained vehicle scheduling and the incorporation of a learning strategy. J. Oper. Res. Soc. Vol.49, pp. $700-708$

[9] Ayed A. Salman, Imtiaz Ahmad, Sabah Al-Madani. 2002. Particle swarm optimization for task assignment problem. Microprocess and Microsyst. Vol.26, pp. 363371.

[10] B. Hu and G.R. Raidl.Effective neighborhood structures for the generalized traveling salesman problem. In J.I. van Hemert, C. Cotta (Eds.), Evolutionary Computation in Combinatorial Optimization-EvoCOP 2008, Vol. 4972 of Lecture Notes in Computer Science, SpringerVerlag, Berlin, Germany. pp. 36-47.

[11]B.Meyer. Hybrids of constructive meta-heuristics and constraint programming. A case study with ACO. In Chr. Blum, M.J.Blesa, A. Roli, and M. Sampels, editors, Hybrid Metaheuristics-An emergent approach for optimization. Springer Verlag, New York, 2008.

[12] Barnes, J. W and M. Laguna. Solve the multiple-machine weighted flow time problem using tabu search. IIE Trans.vol. 25, 1993, pp. 121-128.

[13] Barnes, J. W., V. Wiley, J. Moore, and D. Ryer. 2004. Solving the aerial fleet refueling problem using group theoretic tabu search. Math and Comput Model. pp.6-8.

[14] Battiti, R. and G. Tecchiolli.1994.The Reactive Tabu Search. ORSA J Comput. Vol, 6, pp.126-140.

[15] Bell, J.E., McMullen, P.R. 2004.Ant colony optimization techniques for the vehicle routing problem. Adv. Eng. Inform. Vol. 1, pp. 41-48.

[16] Binato, S., Faria, H. Jr., Resende, M.G.C.2001. Greedy randomized adaptive path relinking. Proceedings of the 4th Metaheuristics International Conference, pp. 393397.
[17] Bullnheimer, B., Hartl, R.F., Strauss, C.1999. An improved ant system algorithm for the vehicle routing problem. Ann. Oper. Res.vol. 89, pp. 319-328.

[18] C. Avanthay, A. Hertz, and N. Zufferey. 2003. A Variable Neighborhood Search for Graph Coloring. Eur. J. Oper. Res. Vol. 151, pp. 379-388.

[19] Christian Blum, Jakob Puchinger, Günther R. Raidl, Andrea Roli. 2011.Hybrid metaheuristics in combinatorial optimization. A survey Appl. Soft. Comput. Vol.11, pp. 4135-4151.

[20] C. Blum, M.J. Blesa. 2009. Solving the KCT problem: large-scale neighborhood search and solution merging. In.E. Alba, C. Blum, P. Isasi, C. León, J.A. Gómez (Eds.), Optimization Techniques for Solving Complex Problems. Hoboken, NJ,Wiley \& Sons. pp. 407-421.

[21] C. C. Ribeiro and M. C. Souza. 2002. Variable neighborhood search for the degree-constrained minimum spanning tree problem. Discret. Appl. Math. vol.118, pp. 43-54.

[22] C. H. Papadimitriou and K. Steiglitz. 1982. Combinatorial Optimization - Algorithms and Complexity Dover Publications, Inc., New York

[23] C. Walshaw. 2004.Multilevel refinement for combinatorial optimization problems. Ann. Oper. Res. Vol.131, pp.325-372.

[24] C. Wilbaut, S. Hanafi, A. Fréville\& S. Balev. 2009. Tabu search: global intensification using dynamic programming. Control Cybern. Vol.35, pp. 579-598.

[25] Carlton, W.and J. W. Barnes. Solving the Traveling Salesman Problem with Time Windows Using Tabu Search. IIE Trans. Vol. 28,1996, pp. 617-629.

[26] Chen, A.L., G.K. Yang and Z.M. Wu. 2006. Hybrid discrete particle swarm optimization algorithm for capacitated vehicle routing problem. J. Zhejiang University Sci. vol. 7, pp.607-614.

[27] Chiung Moon et al. 2002. An efficient GA for the TSP with precedence constraints. Eur. J. Oper. Res. Vol. 140, pp. 606-617.

[28] Chu, P.C. and J.E. Beasley.1998. A Genetic Algorithm for the Multidimensional Knapsack Problem. J Heuristics. Vol. 4, pp. 63-86.

[29] Colorni A., Dorigo M., Maniezzo V. \& Trubian M. 1994. Ant system for job-shop scheduling. Belgian J. Oper. Res. Stat. and Comput. Sci. vol. 34, pp. 39-54.

[30] Consoli S, Darby-Dowman K, Mladenovic N and Moreno J. 2009. Variable neighborhood search for the minimum labelling Steiner tree problem. Ann. Oper. Res. Vol. 172, pp. 71-96.

[31] Consoli, S., Moreno-Perez, JA., Darby-Dowman, K. and Mladenović N. 2010. Discrete particle swarm optimization for the minimum labelling Steiner tree problem. Nat. Comput. Vol. 9 pp. 29- 46.

[32] D. T. Connolly. 1990. An improved annealing scheme for the QAP. Eur. J. Oper. Res. Vol. 46, pp. 93-100.

[33] D.L. Applegate, R.E. Bixby, V. Chvátal \& W.J. Cook. 1998. On the solution of the traveling salesman problem. Doc. Math., Extra Volume ICM III, pp. 645-656. 
[34] Dorigo M and Gambardella LM. Ant colony system: a cooperative learning approach to the traveling salesman problem. IEEE T. Evol. Comput.vol. 1, 1997, pp. 53-66.

[35] Dorigo M, Maniezzo V, Colorni A. 1991. Positive feedback as a search strategy. Technical Report 91-016, Politecnico di Milano, Italy.

[36] Duarte A, Escudero LF, Marti R, Mladenovic N, Pantrigo JJ and Sanchez-Oro J. 2012. Variable Neighborhood Search for the Vertex Separation Problem. Comput. and Oper. Res.

[37] E. Danna, E. Rothberg, C. Le Pape. 2003. Integrating mixed integer programming and local search: a case study on job-shop scheduling problems. In:Fifth International Workshop on Integration of $\mathrm{AI}$ and $\mathrm{OR}$ techniques in Constraint Programming for Combinatorial Optimization Problems (CP-AI-OR'2003), pp. 65-79.

[38] E. J. Teoh, Huajin Tang and K. C Tan. 2006. A Columnar Competitive Model with Simulated Annealing for Solving Combinatorial Optimization Problems. Proc. IEEE Int Jt Conf Neural Netw, Vancouver, BC, Canada, July $16-21$ pp. 3254-3259.

[39] E. Rothberg. 2007. An evolutionary algorithm for polishing mixed integer programming solutions. INFORMS. J. Comput. Vol. 19 pp. 534-541.

[40] E-G. Talbi, O. Roux, C. Fonlupt, and D. Robilliard. 2001. Parallel ant colonies for the quadratic assignment problem. Future. Gener. Comput.Syst. vol. 17,pp. 441449.

[41] F. Glover.1986.Future paths for integer programming and links to artificial intelligence. Comput. \& Oper. Res. Vol. 13, pp. 533-549.

[42] F. Glover and G. Kochenberger. 2003. Handbook of Metaheuristics. Vol. 57 of International Series in Oper. Res. and Manag. Sci, Kluwer Academic Publishers.

[43] F. Glover. 1968. Surrogate constraints, Oper. Res. Vol. $16,741-749$.

[44] Feo, T. A., \& Resende, M. G. C. 1995. Greedy randomized adaptive search procedure. J. Glob. Optim. Vol. 6, pp. 109-133.

[45] Fleurent, C., Glover F. 1999. Improved constructive multistart strategies for the quadratic assignment problem using adaptive memory. INFORMS J. Comput. Vol.11, pp. 198-204.

[46] Frank Neumann, Carsten Witt. 2006. Ant Colony Optimization and the Minimum Spanning Tree Problem. Electron. Colloq. Comput. Complex. (ECCC) 13

[47] G.R. Raidl and H. Feltl. 2004. An improved hybrid genetic algorithm for the generalized assignment problem. In: H.M. Haddadd, et al. (Eds.), Proceedings of the 2003 ACM Symposium on Appl Comput ACM Press, pp. 990-995.

[48] González-Velarde, J. L. and M. Laguna. 2002. Tabu search with simple ejection chains for coloring graphs. Ann. Oper. Res. Vol. 117, pp. 165-174.

[49] H. Tamura, A. Hirahara, I. Hatono, M. Umano. 1994. An approximate solution method for combinatorial optimization. Trans. Soc. Instrum. Control. Eng. Vol. 130 pp. 329-336.
[50] Hansen P and Mladenović N. 1997. Variable neighborhoods search for the p-median. Locat. Sci.Vol. 5, pp.207-226.

[51] Harwig, J., J. W. Barnes, J. T. Moore. 2006. An adaptive tabu search approach for 2-dimensional orthogonal packing problems. Mil. Oper. Res. Vol. 11, pp.5-26.

[52] Hirsch, M.J., Meneses, C.N., Pardalos, P.M., Resende, M.G.C. 2007. Global optimization by continuous GRASP. Optim. Lett. Vol. 1, pp. 201-212.

[53] J. Balakrishnan, C.H. Cheng, D.G. Conway, C.M Lau.2003. A hybrid genetic algorithm for the dynamic plant layout problem. Prod. Econ. Vol. 86, pp. 107-120.

[54] J. Bautista and J. Pereira. 2009. A dynamic programming based heuristic for the assembly line balancing problem. Eur. J. Oper. Res.Vol. 194, pp. 787-794.

[55] J. E. Beasley and P. C. Chu(1996) A genetic algorithm for the set covering problem. Eur. J. Oper. Res. Vol 94, pp. 392-404.

[56] J. H. Holland.1975. Adaptation in natural and artificial systems, The University of Michigan Press, Ann Harbor, MI

[57] J. Kennedy and R. C. Eberhart. 1995. Particle swarm optimization. Proc. of IEEE Int Conf. on Neural Netw Piscataway, NJ, USA, pp. 1942-1948.

[58] J. Lazić, S. Hanafi, N. Mladenović, D. Urošević. 2010 Variable neighborhood decomposition search for $0-1$ mixed integer programs. Comput. and Oper. Res. Vol. 37, pp.1055-1067.

[59] J.C. Beck. 2007. Solution-guided multi-point constructive search for job shop scheduling. J. Artif. Intel. Vol. 29, Res. pp. 49-77.

[60] J.E. Gallardo, C. Cotta and A.J. Fernández. 2007 On the hybridization of memetic algorithms with branch-andbound techniques. IEEE Trans. on Syst. Man. and Cybern.-Part B Vol. 37, pp. 77-83.

[61] James, T., Rego, C., Glover, F. 2009. Multistart tabu search and diversification strategies for the quadratic assignment problem. IEEE Trans. Syst. Man. and Cybern. Part A: Systems and Humans. Vol. 39, pp. 579596.

[62] Jingfa Liu, Yu Zheng, Wenjie Liu. 2009. A Heuristic Simulated Annealing Algorithm for the Circular Packing Problem. Genet. Evol. Comput. pp. 802-805.

[63] Kinney, G., J. W. Barnes, and B. Colletti. 2007. A Reactive Tabu Search algorithm with variable clustering for the Unicost Set Covering Problem. Int. J. Oper. Res. Vol. 2, pp.156-172

[64] Kirkpatrick, S., Gelatt, C.D., Vecchi, M.P. 1983.Optimization by simulated annealing. Sci.Vol. 220 pp. 671-680.

[65] Korycinski, D., M.M. Crawford, J.W. Barnes, and J. Ghosh. 2003. Adaptive feature selection for hyperspectral data analysis using a binary hierarchical classifier and tabu search. Proc. 2003 International Geoscience and Remote Sensing Symposium, Toulouse, France, pp. 297-299. 
[66] KP Wang, L Huang, CG Zhou, W Pang. 2003. Particle swarm optimization for traveling salesman problem. Int. Conf. Mach. Learn. and Cybern.Vol. 3, pp.1583-1585.

[67] Kunpeng KANG. 2012. A Fast Particle Swarm Optimization Algorithm for Large Scale Multidimensional Knapsack Problem. J. Comput. Inform. Syst. Vol. 8, pp.2709-2716.

[68] Kusum Deep and Hadush Mebrathu. 2011. Combined Mutation Operators of Genetic Algorithm for the Travelling Salesman Problem. Int. J. Comb. Optim. Probl. Inform. Vol. 2, pp. 2-24.

[69] L. Shi and S. Ólafsson. 2000. Nested partitions method for global optimization. Oper. Res.Vol. 48, pp. 390-407.

[70] Laguna, M., J. P. Kelly, J. L. González Velarde, and F. Glover. 1995. Tabu search for the multilevel generalized assignment problem. Eur. J. Oper. Res. Vol. 82,pp. 176189.

[71] Laguna, M., J.W. Barnes, F. W. Glover. 1991. Tabu search methods for a single machine scheduling problem. J. Intel. Manuf. Vol. 2, pp. 63-74.

[72] Laguna, M., Martı', R. 1999. GRASP and path relinking for 2-layer straight line crossing minimization. INFORMS. J. Comput. Vol. 11, pp. 44-52.

[73] Lei Yuan, Zhendong Zhao. A Modified Binary Particle Swarm Optimization Algorithm for Permutation Flow Shop Problem. In Proceedings of the Sixth Int Conf Mach Learn Cyberns (ICMLC 2007), Hong Kong (1922) August 2007, pages 902-907.

[74]Lokketangen, A., F. Glover. 1998. Solving zero-one mixed integer programming problems using tabu search. Eur. J. Oper. Res. vol. 106, pp. 624-658.

[75] M. Haouari and J.C. Siala. 2006. A hybrid Lagrangian genetic algorithm for the prize collecting Steiner tree problem. Comput. Oper. Res.vol.33, pp. 1274-1288.

[76] M. Khichane, P. Albert, C. Solnon. Integration of ACO in a constraint programming language. In: Proceedings of ANTS 2008 -6th International Workshop on Ant Colony Optimization and Swarm Intelligence. Vol. 5217 of Lecture Notes in Computer Science, Springer-Verlag, Berlin, Germany, 2008, pp. 84-95.

[77] M. López-Ibáñez and C. Blum. 2010. Beam-ACO for the travelling salesman problem with time windows. Comput. Oper. Res. vol.37, pp. 1570-1583.

[78] M. Lozano and C. García-Martínez. 2010. Hybrid metaheuristics with evolutionary algorithms specializing in intensification and diversification: overview and progress report. Comput. Oper. Res. vol.37, pp. 481-497.

[79] M. Prandtstetter and G.R. Raidl. 2008. An integer linear programming approach and a hybrid variable neighborhood search for the car sequencing problem. Eur. J. Oper. Res. Vol. 191, pp. 1004-1022.

[80] M. Reimann. Guiding ACO by problem relaxation: a case study on the symmetric TSP. In: T. Bartz-Beielstein, M.J. Blesa Aguilera, C. Blum, B. Naujoks, A. Roli, G. Rudolph, M. Sampels (Eds.) Proceedings of HM 2007Fourth International Workshop on Hybrid Metaheuristics, Vol. 4771 of Lecture Notes in Computer Science, Springer-Verlag, Berlin, Germany,2007, pp. $45-55$.
[81] M. Vasquez \& Y.Vimont. 2005. Improved results on the 0-1 multidimensional knapsack problem. Eur. J. Oper. Res. vol. 165, pp. 70-81.

[82] M.G.C. Resende, R. Martí, M. Gallego \& A. Duarte. 2010. GRASP and path relinking for the max-min diversity problem. Comput. Oper. Res. vol.37, pp. 498 508 .

[83] M.J.Geiger, M.Sevaux \& Stefan Voß. 2011 Neighborhood selection in VNS. Metaheuristic international conference $25-28$.

[84] Matheus Rosendo \& Aurora Pozo. 2010. A hybrid Particle Swarm Optimization algorithm for combinatorial optimization problems. IEEE Congr. Evol. Comput. pp. $1-8$.

[85] Mladenovic N, Urosevic D, Perez-Brito D and GarciaGonzalez CG. 2010. Variable neighborhood search for bandwidth reduction. Eur. J. Oper. Res. vol. 200, pp. 1427.

[86] N. Mladenovic and P. Hansen. 1997. Variable Neighborhood Search. Comput. Oper. Res. vol. 24,pp. 1097-1100.

[87] N.Mladenović and D. Urosević. 2001. VNS for the kcardinality tree. In. Proceedings of 4th Metaheuristics International Conference, MIC'2001, Edited by J. Sousa, Porto, Portugal.

[88] Nanry, W. P. and J. W. Barnes. 2000. Solving the pickup and delivery problem with time windows using reactive tabu search. Trans.Res. Part B vol.34, pp.107-121.

[89] Neelam Tyagi and Varshney R.G. 2012. A Model To Study Genetic Algorithm For The Flowshop Scheduling Problem. J. Inform. Oper. Manag. ISSN: 0976-7754 \& E-ISSN: 0976-7762, Volume 3, pp-38-42.

[90] Olli Braysy. 2001. Genetic algorithms for the vehicle routing problem with time windows.Technical Report, 1/2001, University of Vaasa, Vaasa, Finland.

[91] Omar M. Sallabi and Younis El-Haddad. 2009. An Improved Genetic Algorithm to Solve the Travelling Salesman Problem. World Acad. Sci. Eng. and Technol. vol.52, pp. 471-474.

[92] Ou, G., Tamura, H., Tanno, K. and Tang, Z. 2010. A method of solving scheduling problems using an improved guided genetic algorithm. Electron. Comm. Jpn. vol.93, pp. 15-22.

[93] P Shaw. 1998. Using constraint programming and local search methods to solve vehicle routing problems. In: M. Maher, J.-F. Puget (Eds.), Principle and Practice of Constraint Programming - CP98, Vol. 1520 of Lecture Notes in Computer Science, Springer-Verlag, Berlin, Germany, pp. 417-431.

[94] P.C, Chu and J. E. Beasley. 1997. A genetic algorithm for the generalized assignment problem. Comput. Oper. Res. vol.24, pp.17-23.

[95] P. J. M. Van Laarhoven, E. H. L. Aarts, and J. K. Lenstra. 1992. Job Shop Scheduling by Simulated Annealing. Oper. Res. vol.40,pp.113-125.

[96] P.C, Chu \& J.E. Beasley. 1998. A genetic algorithm for the multidimensional knapsack problem. J. Heuristics. vol.4, pp. 63-86. 
[97] PIEROBOM, JL DELGADO, RSB Kaestner, CAA. 2011. Particle Swarm Optimization Applied to task assignment problem. In: $\mathrm{X}$ Brazilian Congr on Comput Intel. Fortaleza, CE: Proceedings of the 2011 CBIC

[98] Prais, M., Ribeiro, C.C. 2000. Reactive GRASP: an application to a matrix decomposition problem in TDMA traffic assignment. INFORMS. J. Comput. vol. 12, pp.164-176.

[99] Puchinger, J. and G. R. Raidl. 2005. Relaxation Guided Variable Neighborhood Search. In Proceedings of the XVIII Mini EURO Conference on VNS, Tenerife, Spain

[100] R. K. Ahuja, J. B. Orlin and A. Tiwari. 2000. A descent genetic algorithm for the quadratic assignment problem. Comput. Oper. Res. vol. 27, pp. 917-934.

[101] R. Montemanni and D.H. Smith. 2010. Heuristic manipulation, tabu search and frequency assignment. Comput. Oper. Res. vol.37, pp.543-551.

[102] R.K. Congram, C.N. Potts, S.L. van de Velde. 2002. An iterated dynasearch algorithm for the single-machine total weighted tardiness scheduling problem. INFORMS J. Comput. vol.14, pp. 52-67.

[103] Ribeiro, C.C., Uchoa, E., Werneck, R.F. 2002. A hybrid GRASP with perturbations for the Steiner problem in graphs. INFORMS. J. Comput. vol.14, pp. 228-246.

[104] S. Pirkwieser, G.R. Raidl, J. Puchinger. Combining Lagrangian decomposition with an evolutionary algorithm for the knapsack constrained maximum spanning tree problem. In: C. Cotta, J.I. van Hemert (Eds.), Evol Comput Comb Optim-EvoCOP 2007, Vol. 4446 of Lecture Notes in Computer Science, SpringerVerlag, Berlin, Germany, 2007, pp. 176-187.

[105] S. Prestwich, S. Tarim, R. Rossi \& B. Hnich. 2009. Evolving parameterized policies for stochastic constraint programming. In: I. Gent (Ed.), Principles and Practice of Constraint Programming-CP 2009, Vol. 5732 of Lecture Notes in Computer Science, Springer-Verlag, Berlin Heidelberg, Germany, pp. 684-691.

[106] S.-M. Tse, Y. Liang, K.-S. Leung, K.-H. Lee, T.S.K. Mok. A memetic algorithm for multiple-drug cancer chemotherapy schedule optimization. IEEE Trans on Syst Man, Cybern-Part B, vol.37, 2007, 84-91.

[107] Scrich, C. R., V. A. Armentano and M. Laguna. 2004. Tardiness minimization on a flexible job shop: a tabu search approach. J. Intel. Manuf. vol.15,pp. 103115 .
[108]Sevkli, M., Aydin,. M.E. A variable neighborhood search implementation for job shop scheduling problems. In Pro. Of EvoCOP 2006 , Lecture Notes in Computer Science 3906, Budapest, Hungary, (10-12 April 2006), pp.261271.

[109]Sevkli, M., Guner, A. R. 2008. A Discrete Particle Swarm Optimization Algorithm for Uncapacitated Facility Location Problem. J. Artif. Evol. Appl. Vol. 2008, pp. 1-9.

[110]Surekha P. 2010. Solving fuzzy based job shop scheduling problems using GA and ACO. J Emerg Trends Comput and Inform Sci, vol.1, pp.95-102.

[111]T Stützle. 2006. Iterated local search for the quadratic assignment problem. Eur. J. Oper. Res. vol.174, pp. $1519-1539$

[112]Tasgetiren MF, Sevkli M, Liang YC, Gencyilmaz G. Particle swarm optimization algorithm for single machine total weighted tardiness problem. In: Proc. of the IEEE Congr Evol Comput, Portland,vol.2, 2004, pp.1412-1419.

[113]Tate David M., Smith Alice E. 1995. A Genetic Approach to the Quadratic Assignment Problem. Comput. Oper. Res. vol. 22, pp.73-83.

[114]Thomas Stutzle. An ant approach to the flow shop problem. In Proceedings of the 6th European Congress on Intelligent Techniques \& Soft Computing (EUFIT'98), Verlag Mainz, Aachen, volume 3, 1998, pp $1560-1564$.

[115]Ulrike Schneider. 2011. A Tabu search tutorial based on a real world scheduling problem. Cent. Eur. J. Oper. Res. vol. 19 , pp. 467-493.

[116]V.J. Rayward-Smith and A.S. Wade. 2000. Effective Local Search for the Steiner Tree Problem. In Advances in Steiner Trees ed. by Ding-Zhu Du, J. M.Smith and J.H. Rubinstein, Kluwer.

[117]Xu Hao. 2010. Optimization Models and Heuristic Method Based on simulated annealing Strategy for Solving Travelling Solution Problem. Appl. Mech. Mater. vols (34-35),pp 1180-1184.

[118]Yannis Marinakis. 2012. Multiple phase neighborhood search GRASP for the capacitated vehicle routing problem. Expert. Syst. Appl. vol. 39, pp. 6807-6815.

[119]Zar Chi Su Su Hlaing, May Aye Khine. 2011. An Ant Colony Optimization Algorithm for Solving Traveling Salesman Problem. Int Proc of Comput Sci Inform Technol, vol.16, pp.54-59. 